\title{
The Assessment of Cerebrovascular Response to a Language Task from the Addenbrooke's Cognitive Examination in Cognitive Impairment: A Feasibility Functional Transcranial Doppler Ultrasonography Study
}

\author{
Lucy C. Beishon ${ }^{\mathrm{a}, *}$, Ronney B. Panerai ${ }^{\mathrm{a}, \mathrm{b}}$, Thompson G. Robinson ${ }^{\mathrm{a}, \mathrm{b}}$, Hari Subramaniam ${ }^{\mathrm{a}, \mathrm{c}}$ \\ and Victoria J. Haunton ${ }^{\mathrm{a}, \mathrm{b}}$ \\ ${ }^{a}$ Department of Cardiovascular Sciences, University of Leicester, Leicester, UK \\ ${ }^{\mathrm{b}}$ NIHR Leicester Biomedical Research Centre, British Heart Foundation Cardiovascular \\ Research Centre, Glenfield Hospital, Leicester, UK \\ ${ }^{\mathrm{c}}$ Leicestershire Partnership Trust, Evington Centre, Gwendolen Road, Leicester, UK
}

Accepted 14 August 2018

\begin{abstract}
.
Background: The incidence of dementia is predicted to rise rapidly, but sensitive diagnostic tests remain elusive. Changes in cerebral blood flow velocity $(\mathrm{CBFv})$ can occur at an early stage of cognitive decline, and can be measured by transcranial Doppler ultrasonography (TCD).

Objective: The aim of this study was to characterize the CBFv changes that occur in healthy older adults (HC), mild cognitive impairment (MCI), and Alzheimer's disease (AD), in response to a language task from the Addenbrooke's cognitive examination (ACE-III).

Methods: Participants underwent bilateral TCD, continuous heart rate (ECG), end-tidal $\mathrm{CO}_{2}$ (capnography, $\mathrm{ETCO}_{2}$ ), and beat-to-beat blood pressure (Finometer, MAP), monitoring, during a 5-minute baseline, followed by cognitive tasks from the ACE-III. Data are presented for a language task (repeating words and phrases aloud), as peak percentage change in CBFv, $\mathrm{HR}, \mathrm{MAP}$, and $\mathrm{ETCO}_{2}$ from a normalized baseline.

Results: 30 participants (mean age 73.2 years, $20 \%$ female) were recruited; $\mathrm{HC}(n=10)$, MCI $(n=10)$, AD $(n=10)$. Language scores did not differ between groups $(p=0.16)$. Peak percentage change in CBFv differed between groups with the language task (HC: 15.9 (7.5)\%, MCI: 6.7 (4.5)\%, AD: 0.1 (7.1)\%; $p<0.005)$. However, changes in MAP (HC: 7.9 (4.6)\%, MCI: -0.1 (0.9)\%, AD: 0.9 (4.4)\%; $p<0.005)$, HR (HC: 8.8 (8.2)\%, MCI: 0.7 (4.3)\%, AD: -0.5 (5.6)\%; $p=0.005)$, and $\mathrm{ETCO}_{2}(\mathrm{HC}$ -0.9 (3.2)\%, MCI: $0.9(3.2) \%$, AD: $-5.2(5.7) \% ; p=0.006)$, also occurred.

Conclusions: TCD measured $\mathrm{CBFv}$ changes to a language task from the ACE-III was feasible in a cognitively impaired population, further work is required in a larger population.
\end{abstract}

Keywords: Alzheimer's disease, cerebral blood flow, dementia imaging, mild cognitive impairment

\footnotetext{
*Correspondence to: Lucy Beishon, Department of Cardiovascular Sciences, College of Life Sciences, University of Leicester, Level 2, Clinical Sciences Building, Leicester Royal Infirmary,
} 


\section{INTRODUCTION}

The world prevalence of dementia is forecast to reach 74.7 million by 2030 , doubling every 20 years [1]. Therefore, there is a pressing need for the development of sensitive diagnostic tools to detect the very earliest stages of dementia and facilitate the timely delivery of emerging therapeutics. Mild cognitive impairment (MCI) describes an early stage of cognitive decline, lying on a continuum between normal aging and dementia [2]. Importantly, patients with MCI remain functionally independent, thus providing an early opportunity for potential disease modifying treatments [2]. However, not all patients with MCI will develop dementia [2]. Therefore, a sensitive test to not only diagnose MCI but to also predict those who will convert to dementia would be valuable in instigating early treatments for those at greatest risk.

In recent years, impaired cerebral blood flow (CBF) has been identified as an important pathological mechanism in both Alzheimer's disease (AD) and vascular dementia (VaD) [3, 4]. It has become increasingly recognized that perfusional deficits can both precede and exacerbate the deposition of tau and amyloid in $\mathrm{AD}$ [5]. A recent systematic review demonstrated abnormalities in cerebral hemodynamics at the MCI stage, and thus could provide an early biomarker of cognitive decline [6]. Consistent across studies, those with MCI and dementia tend to have lower cerebral perfusion, reduced cerebral vasoreactivity, and higher measures of vessel resistance [6-9].

Transcranial Doppler ultrasonography (TCD) is a non-invasive, ultrasound-based technique to measure $\mathrm{CBF}$ velocity (CBFv) in the intra- and extra-cranial arteries $[10,11]$. TCD can be used to measure resting $\mathrm{CBFv}$, and changes in $\mathrm{CBFv}$ in response to task activation [11-14]. Cerebral autoregulation is the process by which the cerebral cortex maintains a constant blood supply, despite fluctuations in blood pressure, and $\mathrm{CO}_{2}$. Neurovascular coupling (NVC) describes the important relationship between neuronal stimulation and perfusion; whereby increasing neuronal activation results in increased cerebral perfusion to match increasing cortical metabolism [15]. NVC is achieved through three mechanisms; metabolic, myogenic, and autonomic. Metabolic regulation is achieved through the dilation of vessels in response to vasoactive mediator release (i.e., nitric oxide), at times of high metabolic activity, resulting increased flow, and thus supply of nutrients and removal of waste products. Secondly, myogenic regulation is achieved through relaxation and contraction of the vessel smooth muscle in response to changes in transmural pressure, resulting in vasodilation and constriction respectively, protecting the brain from sudden rises or falls in pressure. Thirdly, autonomic regulation through changes in sympathetic tone can adjust vessel constriction, and thus flow. TCD can be used to evaluate the integrity of the NVC process by measuring changes in $\mathrm{CBFv}$ in response to neuronal stimulation $[13,15]$. TCD is particularly advantageous in people with cognitive impairment, as it can be used in older adults with metal implants or pacemakers, and can be more acceptable than CT or MRI scans [11].

Only a few TCD studies have investigated neuroactivation or cognitive stimulation in dementia, with mixed results [16-18]. No TCD studies have investigated the changes in $\mathrm{CBFv}$ that occur in response to stimulation from a language task from a cognitive assessment tool in cognitive impairment. Language deficits are known to occur in the early stages of AD, particularly in verbal fluency, lexical retrieval, and comprehension of higher order written and spoken language [19], and thus could be used to investigate deficits in NVC.

The primary aim of this study was therefore to assess the feasibility of using a language task from the Addenbrooke's cognitive examination (ACE-III) in three different groups; healthy older adults, MCI, and $\mathrm{AD}$. We specifically chose a language task from a cognitive assessment tool that is in widespread clinical practice, and used for the diagnosis of both MCI and dementia. The primary outcome for this study was to assess the feasibility of a TCD-measured language task activation in a cognitively impaired population. Secondary outcomes were: 1) to quantify the peak percentage change in $\mathrm{CBFv}$ in the dominant and non-dominant hemispheres, and mean arterial pressure (MAP), heart rate (HR), and end-tidal $\mathrm{CO}_{2}$ $\left(\mathrm{ETCO}_{2}\right)$ across the three groups described above; 2) to investigate for correlations between cognitive test score and $\mathrm{CBFv}$ response within the language domain; 3) to examine for a difference in hemispheric lateralization of peak $\mathrm{CBFv}$ changes between the three groups outlined above.

\section{METHODS}

This was a feasibility case-controlled study undertaken between May and November 2017. The study had research ethics committee approval (reference: 17/WA/0089, IRAS 203853). Healthy older adults; 
and adults with a diagnosis of $\mathrm{MCI}$ or $\mathrm{AD}$ were recruited from outpatient memory, geriatric and stroke clinics at the University Hospitals of Leicester NHS Trust and Leicestershire Partnership Trust, and from Join Dementia Research. In addition, healthy volunteers were recruited through poster advertisement at outpatient and university settings, and from family members or enrolled participants. Healthy volunteers were recruited separately to previous studies and are a distinct population. All participants provide written informed consent and the study was carried out according to the Declaration of Helsinki. A sample size calculation was not performed at this stage as the primary outcome for the study was feasibility and acceptability of the protocol in a cognitively impaired population, with secondary aims to assess changes in hemodynamic parameters, without pre-established effect sizes.

Inclusion criteria for the study were: aged over 18 years, able to comply with study requirements, good understanding of written and verbal English. Participants with a diagnosis of MCI or AD were classified at a consensus meeting according to the NIA/AA 2011 guidelines and using clinical characteristics and relevant brain imaging. Healthy older adults (HC) were suitable for inclusion provided there was no evidence of subjective or objectively assessed or diagnosed cognitive deficit, and no major medical co-morbidity or medication use that could adversely affect cognition. Study specific exclusion criteria were: aged under 18 years, unable to comply with study requirements, pregnancy, planning pregnancy or lactating, severe carotid artery stenosis, atrial fibrillation, severe cardiac failure (ejection fraction $<20 \%$ ), severe respiratory disease, and inability to consent to the study. Participants on anti-dementia drugs (acetylcholinesterase inhibitors (AChEI), NMDA receptor antagonists) were not excluded from this study and medications were not withheld.

\section{Baseline demographics}

55 participants were recruited to the study, of whom twenty-five were excluded due to: inadequate temporal windows $(n=13)$, visual impairment $(n=1)$, VaD diagnosis $(n=3)$, un-matched $(n=5)$ and misclassification $(n=3)$. There were no significant differences in age or sex of the excluded participants from those analyzed in the study. Mean age of the cohort was 73.2 (7.0) years, six (20\%) were female, 29 (96.7\%) Caucasian, and five (16.7\%) were lefthanded.
Baseline characteristics are listed for the total cohort and sub-groups in Table 1. Hypertension was more prevalent in the MCI group $(60 \%, p=0.036)$, and greater AChEI use was seen in the AD group $(90 \%, p<0.005)$. No other significant between-group differences were seen in demographic data (Table 1).

\section{Study procedure}

Volunteers who met the above criteria were invited to the Cerebral Haemodynamics in Ageing and Stroke Medicine Laboratory. Participants were requested to refrain from caffeine, alcohol, heavy meals, or strenuous exercise for at least $4 \mathrm{~h}$ prior to study commencement. All study procedures were undertaken in a quiet, temperature-controlled laboratory, free from distraction. Participants provided baseline information on age, sex, comorbidities, and medication use, or these were accessed from medical records. Handedness was assessed using the Edinburgh Handedness Inventory [20]. Right- and left-handed volunteers were suitable for study inclusion. Participants underwent continuous recording of bilateral CBFv (Viasys Companion III or DWL Doppler box) in the middle cerebral arteries (MCA), HR (3-lead ECG), continuous blood pressure (arterial volume clamping on non-dominant hand, Finometer, Finapres Medical Systems; Amsterdam, The Netherlands), and end-tidal $\mathrm{CO}_{2}\left(\mathrm{ETCO}_{2}\right.$, Capnography with nasal cannulae, Capnocheck Plus). TCD probes were secured in place using a headframe. Signals were sampled at 500 samples/s and stored in the data acquisition system (PHYSIDAS, Dept Medical Physics, University Hospitals of Leicester NHS Trust).

Initially, a 5-min baseline recording was taken at rest, followed by all tasks from the ACE-III, in the order that they would be undertaken in the clinical setting. There was a 1-min baseline between cognitive tasks to allow $\mathrm{CBFv}$ to return to baseline. The questions were divided into 3 sections and the equipment was paused between recordings to allow auto-calibration of the Finometer. The Finometer was calibrated prior to each recording using the brachial blood pressure. Question timings were marked manually with an event recorder. Each task was performed with one trial. The total assessment, including baseline data collection was approximately $2 \mathrm{~h}$. A similar protocol has been published previously in a healthy cohort [21, 22].

Data were analyzed offline using software developed by this group previously [13, 23]. Data were visually inspected for large non-physiological spikes 
Table 1

Baseline demographics of participants enrolled in the study. Data are number (percentage) for nominal data, and mean (standard deviation) or median [inter-quartile range] for continuous data. Significance testing by chi-square, one-way analysis of variance or Kruskall-Wallis

\begin{tabular}{|c|c|c|c|c|c|c|}
\hline Demographic & $\mathrm{n}$ & ALL & $\begin{array}{l}\text { Healthy } \\
\text { Controls }\end{array}$ & $\begin{array}{c}\text { Mild } \\
\text { cognitive } \\
\text { impairment }\end{array}$ & $\begin{array}{l}\text { Alzheimer's } \\
\text { disease }\end{array}$ & $p$ \\
\hline $\mathrm{n}$ & & 30 & 10 & 10 & 10 & \\
\hline Female sex $(n, \%)$ & 30 & $6(20)$ & $2(20)$ & $2(20)$ & $2(20)$ & 1.00 \\
\hline Age (y) & 30 & $73.2(7.0)$ & $71.4(5.7)$ & $73.3(6.4)$ & $74.9(8.7)$ & 0.55 \\
\hline Caucasian $(\mathrm{n}, \%)$ & 30 & $29(96.7)$ & $10(100)$ & $10(100)$ & $9(90)$ & 0.36 \\
\hline Left Handedness (n, \%) & 30 & $5(16.7)$ & $1(10)$ & $3(30)$ & $1(10)$ & 0.38 \\
\hline Age at completion of education $(\mathrm{y})$ & & $16[3.8]$ & $18[5.5]$ & $15[3]$ & $16[4.3]$ & 0.15 \\
\hline Body mass index & 29 & $25.2(4.2)$ & $25.8(3.2)$ & $25.4(5.8)$ & $24.2(3.1)$ & 0.70 \\
\hline \multicolumn{7}{|l|}{ Smoker } \\
\hline Current (n, \%) & 30 & $2(6.7)$ & $0(0)$ & $2(20)$ & $0(0)$ & 0.17 \\
\hline $\operatorname{Ex}(\mathrm{n}, \%)$ & & $15(50)$ & $4(40)$ & $6(60)$ & $5(50)$ & \\
\hline Alcohol intake $>14$ units/week (n, \%) & 30 & $5(16.7)$ & $2(20)$ & $2(20)$ & $1(10)$ & 0.79 \\
\hline Diabetes $(\mathrm{n}, \%)$ & 30 & $1(3.3)$ & $0(0)$ & $1(10)$ & $0(0)$ & 0.36 \\
\hline Hypertension (n, \%) & 30 & $9(30)$ & $2(20)$ & $6(60)$ & $1(10)$ & 0.036 \\
\hline Hypercholesterolemia (n, \%) & 30 & $11(36.7)$ & $2(20)$ & $6(60)$ & $3(30)$ & 0.16 \\
\hline Cardiovascular disease $(\mathrm{n}, \%)$ & 30 & $2(6.7)$ & $0(0)$ & $1(10)$ & $1(10)$ & 0.59 \\
\hline Previous stroke (n, \%) & 30 & $3(10)$ & $0(0)$ & $2(20)$ & $1(10)$ & 0.33 \\
\hline Depression $(\mathrm{n}, \%)$ & 30 & $2(6.7)$ & $0(0)$ & $1(10)$ & $1(10)$ & 0.59 \\
\hline Antidepressant use (n, \%) & 30 & $3(10)$ & $0(0)$ & $2(20)$ & $1(10)$ & 0.33 \\
\hline Acetylcholinesterase inhibitor use (n, \%) & 30 & $12(40)$ & $0(0)$ & $3(30)$ & $9(90)$ & $<0.005$ \\
\hline Antihypertensive use (n, \%) & 30 & $8(26.7)$ & $1(10)$ & $5(50)$ & $2(20)$ & 0.11 \\
\hline ACE-III total score & 30 & $87[17]$ & $96.5[3.0]$ & $87[7.3]$ & $77.5[9.5]$ & $<0.005$ \\
\hline ACE-III Language score & 30 & $4[0]$ & $4[0]$ & $4[0]$ & $4[0]$ & 0.38 \\
\hline
\end{tabular}

in $\operatorname{CBFv}(<100 \mathrm{~ms})$, which were removed by linear interpolation. A median filter was applied to the CBFv signal to remove smaller spikes and all data were low-pass filtered at $20 \mathrm{~Hz}$ with a zerophase Butterworth filter. Beat-to-beat mean CBFv, mean arterial pressure (MAP), $\mathrm{ETCO}_{2}$, and HR were determined by the R-R interval (3-lead ECG). Data underwent standard polynomial interpolation and then re-sampling at $5 \mathrm{~Hz}$ in order to generate signals with a uniform time base [13, 23].

Signals were synchronized using the event marker, and population mean responses were calculated. Data are presented for the dominant and nondominant hemispheres as peak percentage change in $\mathrm{CBFv}, \mathrm{MAP}, \mathrm{HR}$, and $\mathrm{ETCO}_{2}$ from a 20-s baseline prior to task initiation (T1). Two time points were selected to measure peak activation. Firstly, T2: the initial response to stimulation occurring $5 \mathrm{~s}$ after task initiation (mean peak percentage change in CBFv occurring at 25-30 s). Secondly, T3: the sustained response, occurring at $15 \mathrm{~s}$ after task initiation (mean peak percentage change in $\mathrm{CBFv}$ occurring at $30-40 \mathrm{~s})$. This analysis has been published previously in a young, healthy cohort $[21,22]$. In this communication of the initial results of the new approach, of coupling the traditional ACE-III assessment of patients with $\mathrm{MCI}$ and $\mathrm{AD}$ with NVC examination using TCD, we report only on one task: repeating words and phrases aloud. The reasons for this choice will be discussed below.

The dominant hemisphere was considered right side for left-handed individuals and left side for right-handed individuals, with the converse for the non-dominant hemispheres. The data were combined for the right and left hemispheres (left- and righthand dominant, respectively) to produce a dataset for changes occurring in the dominant and non-dominant hemispheres as opposed to left- or right-hand dominance.

\section{Statistical analysis}

Data were tested for normality prior to analysis using the Shapiro-Wilks test. Continuous, normally distributed data are presented as mean (standard deviation), and non-normally distributed as median [inter-quartile range]. Nominal data are presented as number (percentage), with significance testing by chi-square analysis. Testing for statistical significance was by one-way analysis of variance (continuous, normally distributed), or Kruskall-Wallis (continuous, non-parametric). For 
Table 2

Baseline parameters for healthy control, Alzheimer's disease, and mild cognitive impairment groups. $\mathrm{CBFv}$, cerebral blood flow velocity; MAP, mean arterial pressure; $\mathrm{ETCO}_{2}$, end-tidal $\mathrm{CO}_{2}$. Data are presented as mean (standard deviation). Significance testing by one-way ANOVA

\begin{tabular}{lcccc}
\hline Parameter & $\begin{array}{c}\text { Healthy } \\
\text { controls }\end{array}$ & $\begin{array}{c}\text { Alzheimer's } \\
\text { disease }\end{array}$ & $\begin{array}{c}\text { Mild } \\
\text { cognitive } \\
\text { impairment }\end{array}$ & $p$ \\
\hline $\mathrm{n}$ & 10 & 10 & 10 & \\
$\mathrm{CBFv}$ non-dominant $(\mathrm{cm} / \mathrm{s})$ & $44.4(9.4)$ & $42.7(7.3)$ & $46.2(7.8)$ & 0.64 \\
$\mathrm{CBFv}$ dominant $(\mathrm{cm} / \mathrm{s})$ & $45.7(7.1)$ & $42.8(8.0)$ & $45.0(8.1)$ & 0.69 \\
$\mathrm{MAP}(\mathrm{mmHg})$ & $98.2(15.0)$ & $85.2(29.7)$ & $91.7(13.6)$ & 0.39 \\
$\mathrm{Heart} \mathrm{rate}(\mathrm{bpm})$ & $64.8(12.7)$ & $64.3(13.5)$ & $66.6(7.2)$ & 0.90 \\
$\mathrm{ETCO}_{2}(\mathrm{mmHg})$ & $37.8(2.7)$ & $35.9(2.8)$ & $37.8(2.9)$ & 0.24 \\
\hline
\end{tabular}

non-parametric data, correlation analysis was performed using Spearman correlation. To investigate for the effect of hemispheric dominance and for an interaction between patient group and dominance, a two-way mixed ANOVA was used. Where an interaction effect was present, a univariate analysis was undertaken at all levels of the dependent and independent variable to identify main effects. Where a main effect was present for dominance, this was further investigated using paired t-testing within patient groups. Post hoc testing was by Bonferroni correction or Tukey for multiple comparisons as appropriate. Analyses were considered significant where $p<0.05$. Data analyses were performed using SPSS version 22 for Windows. Graphs were produced using GraphPad Prism Version 7.0 for Windows.

\section{RESULTS}

\section{Baseline data}

There were no significant differences in $\mathrm{CBFv}$ in the dominant and non-dominant hemispheres between groups at baseline (Table 2). Similarly, there were no differences in peripheral measurements of MAP, HR, or $\mathrm{ETCO}_{2}$ at baseline (Table 2).

\section{Changes in peripheral parameters during cognitive testing}

\section{Heart rate}

A significantly lower peak percentage change in $\mathrm{HR}$ in both MCI and AD participants compared to $\mathrm{HC}$ at T2 (HC: 8.8 (8.2)\%, MCI: 0.7 (4.3)\%, AD: -0.5 (5.6)\%; $p=0.005$ ) (Table 3, Fig. 1). HR differences between groups were not seen at T3 (Table 3, Fig. 1).

\section{Mean arterial pressure}

At T2, significantly lower peak percentage changes in MAP were seen between groups (HC: 7.9 (4.6)\%, MCI: -0.1 (2.9)\%, AD: 0.9 (4.4)\%; $p<0.005)$ (Table 3, Fig. 1). These differences were significant between $\mathrm{HC}$, and both $\mathrm{MCI}$ and $\mathrm{AD}$ groups on posthoc testing (Table 3, Fig. 1). There were no significant differences between groups at T3 (Table 3, Fig. 1).

\section{End-tidal $\mathrm{CO}_{2}$}

At T2, $\mathrm{ETCO}_{2}$ differed between groups (HC: -0.9 (3.2)\%, MCI: $0.9(3.2) \%, \mathrm{AD}:-5.2(5.7) \% ; p=0.006)$ (Table 3, Fig. 1). There was a significantly large negative change in the $\mathrm{AD}$ group; changes were statistically different on post-hoc testing between $\mathrm{AD}$ and HC/MCI groups (Table 3, Fig. 1). At T3, there were no differences between groups in $\mathrm{ETCO}_{2}$ responses (Table 3, Fig. 1).

\section{Changes in cerebral hemodynamic parameters during cognitive testing}

\section{Temporal changes}

Temporal changes in $\mathrm{CBFv}$ occurring in response to a language task in both non-dominant and dominant hemispheres are shown in Fig. 2.

\section{Changes at $T 2$}

Peak percentage change in $\mathrm{CBFv}$ differed between groups in the non-dominant hemisphere (HC: 15.9 (7.5)\%, MCI: 6.7 (4.5)\%, AD: $0.1(7.1) \% ; p<0.005)$, and dominant hemisphere (HC: 16.0 (7.5)\%, MCI: $6.0(4.4) \%$, AD: $-0.6(8.4) \%$; $p<0.005)$ (Table 3, Fig. 2). There was a significantly larger response in the $\mathrm{HC}$ group on post-hoc testing between $\mathrm{HC}$ and both MCI/AD groups (Table 3, Fig. 2). 
Table 3

Parameters at $\mathrm{T} 2$ and T3. Data shown are peak percentage change in cerebral blood flow velocity $(\mathrm{CBFv})$, mean arterial pressure (MAP), heart rate and end-tidal $\mathrm{CO}_{2}\left(\mathrm{ETCO}_{2}\right)$ from a 20 -s baseline, in healthy control, mild cognitive impairment, and Alzheimer's dementia groups. Statistical testing by one-way ANOVA with post-hoc testing by Tukey

\begin{tabular}{|c|c|c|c|c|c|}
\hline Parameter & $\mathrm{n}$ & $\begin{array}{l}\text { Healthy } \\
\text { controls }\end{array}$ & $\begin{array}{l}\text { Alzheimer's } \\
\text { disease }\end{array}$ & $\begin{array}{c}\text { Mild } \\
\text { cognitive } \\
\text { impairment }\end{array}$ & $p$ \\
\hline $\mathrm{n}$ & & 10 & 10 & 10 & \\
\hline \multicolumn{6}{|c|}{ CBFv Non-dominant } \\
\hline $\mathrm{T} 2$ & 30 & $15.9(7.5)$ & $0.1(7.1)$ & $6.7(4.5)$ & $<0.005$ \\
\hline $\mathrm{T} 3$ & 30 & $9.4(8.8)$ & $1.9(8.3)$ & $9.4(7.3)$ & 0.08 \\
\hline \multicolumn{6}{|c|}{ CBFv Dominant } \\
\hline $\mathrm{T} 2$ & 30 & $16.0(7.5)$ & $-0.6(8.4)$ & $6.0(4.4)$ & $<0.005$ \\
\hline $\mathrm{T} 3$ & 30 & $9.4(10.0)$ & $-1.5(5.8)$ & $9.0(9.2)$ & 0.012 \\
\hline \multicolumn{6}{|l|}{ Heart rate } \\
\hline $\mathrm{T} 2$ & 30 & $8.8(8.2)$ & $-0.5(5.6)$ & $0.7(4.3)$ & 0.005 \\
\hline $\mathrm{T} 3$ & 30 & $7.3(5.8)$ & $2.1(7.2)$ & $1.6(4.8)$ & 0.08 \\
\hline \multicolumn{6}{|l|}{ MAP } \\
\hline $\mathrm{T} 2$ & 30 & $7.9(4.6)$ & $0.9(4.4)$ & $-0.1(2.9)$ & $<0.005$ \\
\hline $\mathrm{T} 3$ & 30 & $6.1(4.0)$ & $2.3(5.1)$ & $3.7(4.0)$ & 0.16 \\
\hline \multicolumn{6}{|l|}{$\mathrm{ETCO}_{2}$} \\
\hline $\mathrm{T} 2$ & 30 & $-0.9(3.2)$ & $-5.2(5.7)$ & $0.9(3.2)$ & 0.006 \\
\hline $\mathrm{T} 3$ & 30 & $-3.0(7.8)$ & $-5.5(6.5)$ & $-1.9(2.5)$ & 0.39 \\
\hline
\end{tabular}
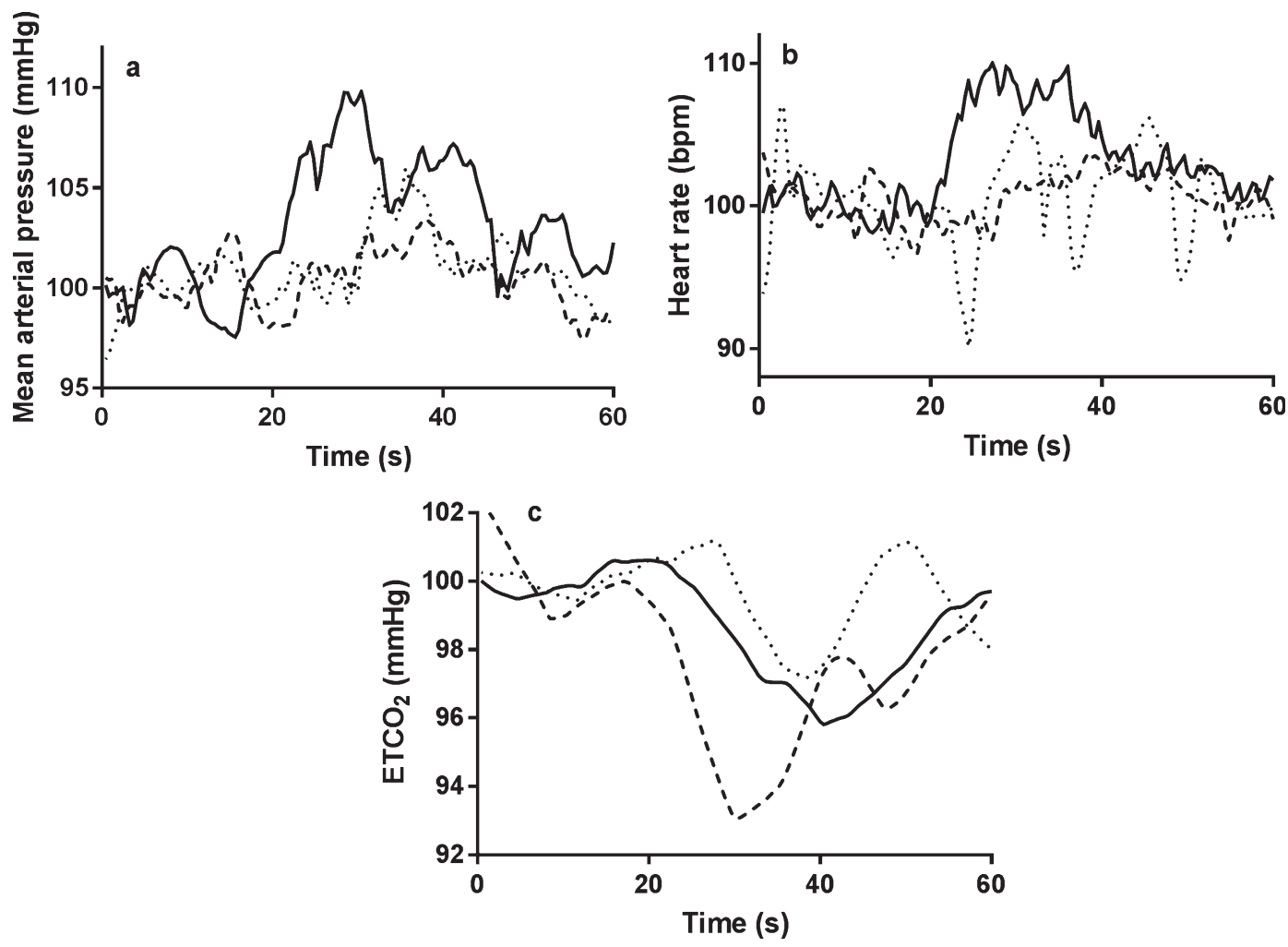

Fig. 1. The temporal changes in MAP (a), heart rate (b), and $\mathrm{ETCO}_{2}$ (c), to the language task. All signals were normalized by baseline values. Continuous line $=$ healthy control, dotted line $=$ mild cognitive impairment, interrupted line = Alzheimer's disease. a) non-dominant hemisphere; b) dominant hemisphere. Task initiation occurred at $20 \mathrm{~s}$. 

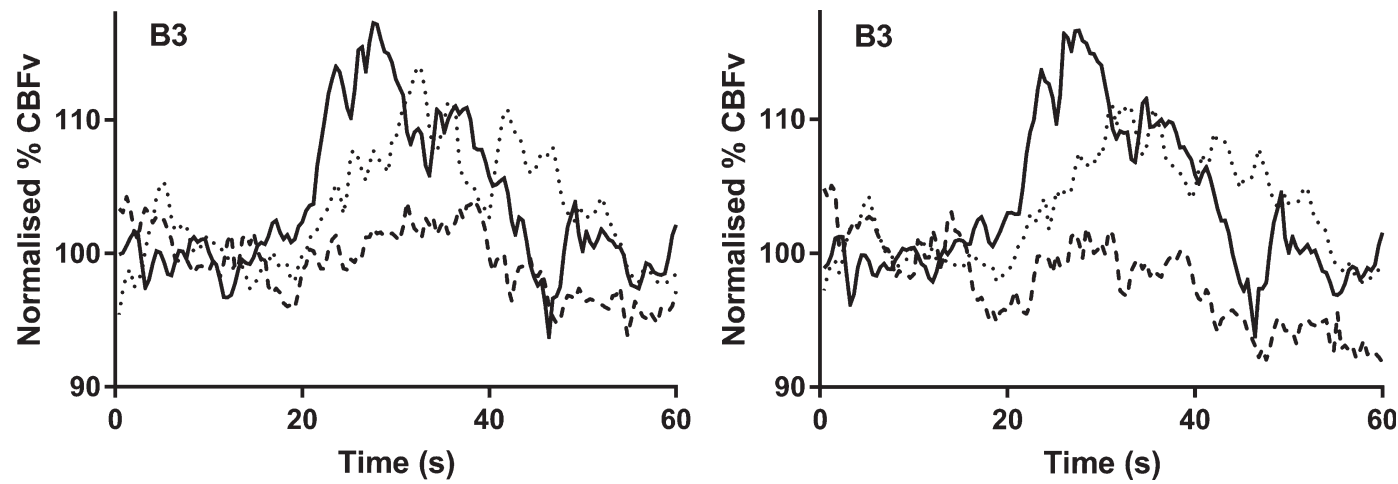

Fig. 2. The temporal changes in cerebral blood flow velocity (normalized \% CBFv) to the language task. All signals were normalized by baseline values. Continuous line $=$ healthy control, dotted line $=$ mild cognitive impairment. interrupted line $=$ Alzheimer's disease. a) non-dominant hemisphere; b) dominant hemisphere. Task initiation occurred at $20 \mathrm{~s}$.

Table 4

Correlation between cognitive test score and peak percentage change in cerebral blood flow velocity (CBFv), for all participants (healthy control, mild cognitive impairment, and Alzheimer's dementia groups combined). Statistical testing by Spearman correlation, with Bonferroni correction for repeated measures

\begin{tabular}{|c|c|c|c|c|c|c|c|}
\hline Parameter & $\mathrm{n}$ & $\begin{array}{c}\text { Correlation } \\
\text { coefficient }\end{array}$ & $p$ & Parameter & $\mathrm{n}$ & $\begin{array}{l}\text { Correlation } \\
\text { coefficient }\end{array}$ & $\bar{p}$ \\
\hline Total ACE- III score & & & & Total ACE- III score & & & \\
\hline CBFv Non-dominant T2 & 30 & 0.44 & 0.028 & CBFv Non-dominant T3 & 30 & 0.27 & 0.30 \\
\hline CBFv Dominant T2 & 30 & 0.40 & 0.06 & CBFv Dominant T3 & 30 & 0.32 & 0.16 \\
\hline ACE-II Language score & & & & ACE-III Language score & & & \\
\hline CBFv Non-dominant $\mathrm{T} 2$ & 30 & 0.22 & 0.48 & CBFv Non-dominant $\mathrm{T} 3$ & 30 & 0.10 & 1.0 \\
\hline CBFv Dominant T2 & 30 & 0.32 & 0.18 & CBFv Dominant T3 & 30 & 0.07 & 1.0 \\
\hline
\end{tabular}
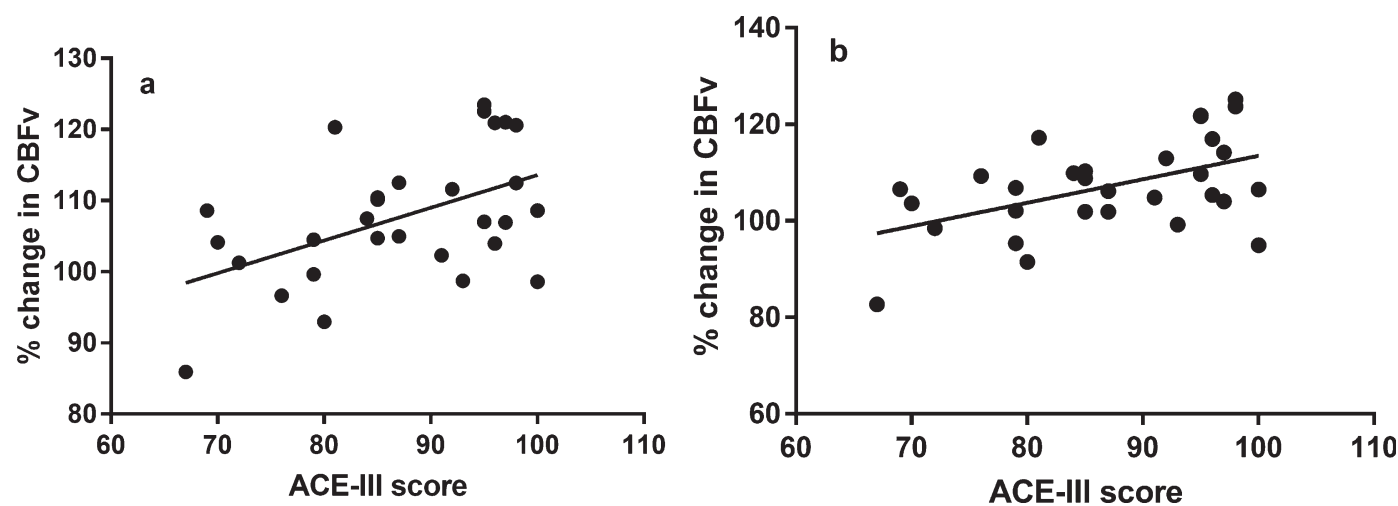

Fig. 3. Positive correlations between normalized percentage change in cerebral blood flow velocity (CBFv) and Addenbrooke's Cognitive Examination III (ACE-III) score, $a=$ non-dominant hemisphere, $b=$ dominant hemisphere. Correlations by Spearman testing.

\section{Changes at T3}

At T3, only the dominant hemisphere showed significant differences between groups (HC: 9.4 (10.0)\%, MCI: 9.0 (9.2)\%, AD: -1.5 (5.8)\%; $p=0.012$ ); significant between $\mathrm{AD}$ and $\mathrm{HC} / \mathrm{MCI}$ groups on post-hoc testing (Table 3, Fig. 2).

\section{Cognitive test score correlation analysis}

Significant, positive correlations were present between peak percentage $\mathrm{CBFv}$ change and total ACE-III score for the whole cohort (HC, MCI, and $\mathrm{AD}$ combined) for non-dominant hemisphere and 
language task response at T2 (Table 4, Fig. 3). There were no significant correlations between total ACEIII score and the language task with peak percentage CBFv change at T3 (Table 4, Fig. 2). There were no significant correlations between language task score and peak percentage change in $\mathrm{CBFv}$ response (Table 4, Fig. 3).

\section{Hemispheric dominance analysis}

There were no statistically significant effects of dominance or interaction between patient group and dominance for $\mathrm{CBFv}$ change to the language task on 2-way mixed ANOVA. In a further analysis, left-handed participants were removed, and data were re-analyzed just for right-handed participants $(n=25)$. There were no differences in baseline CBFv or peripheral parameter data, nor in peak percentage change. The only difference in statistical significance was that the correlation of non-dominant $\mathrm{CBFv}$ response at T2 with total ACE-III score became non-significant $(p=0.07)$.

\section{DISCUSSION}

\section{Main findings}

In summary, the use of a language task from the ACE-III to stimulate CBFv responses was both feasible and acceptable in healthy older adults, MCI, and AD. The protocol was well tolerated and acceptable, and good quality bilateral data were obtained for central and peripheral parameters in 30 participants. Although a power calculation was not performed, peak $\mathrm{CBFv}$ response to a language task showed significant differences in peak percentage change in $\mathrm{CBFv}$ between groups, however, this varied between hemispheres and between time points (i.e., T2 or T3). In addition, where these differences were present, they were also accompanied by changes in the peripheral parameters (MAP, HR, or $\mathrm{ETCO}_{2}$ ). At T2, it is notable that the larger rise in $\mathrm{CBFv}$ was accompanied by a larger rise in both $\mathrm{HR}$ and MAP in the $\mathrm{HC}$ group, and a larger negative change in $\mathrm{ETCO}_{2}$ in the $\mathrm{AD}$ group, which could account for the differences in CBFv change seen across the groups. Furthermore, there was a significantly lower stimulation in $\mathrm{CBFV}$ with the language task in the dominant hemisphere in the $\mathrm{AD}$ group at $\mathrm{T} 3$, which was not accompanied by any changes in $\mathrm{HR}$, MAP, or $\mathrm{ETCO}_{2}$. Therefore, the relationships between stimulation, changes in MAP, $\mathrm{HR}$, and $\mathrm{ETCO}_{2}$ are complex in interpreting their relative contributions to the $\mathrm{CBFv}$ change seen with task activation. There was significant correlation between $\mathrm{CBFv}$ change to the language task and total ACE-III score, but not within the language domain score, and only at T2. Finally, there was no effect of hemispheric dominance on the $\mathrm{CBFv}$ response to the language task.

\section{Task selection}

In previous communications, we described $\mathrm{CBFv}$ responses to the complete set of 20 ACE-III tasks in healthy controls $[22,24]$. When examining the feasibility and sensitivity of this approach in patient populations however, we elected to focus on a single paradigm (repeating words and phrases aloud). There were several reasons for this. Firstly, it would be extremely difficult to report on all 20 paradigms with the same level of detail as in this paper, specifically describing the concomitant changes in systemic variables, including MAP, HR, and $\mathrm{ETCO}_{2}$, that could influence the amplitude and temporal pattern of the CBFv response. Secondly, we purposely selected one of the tasks where the ACE-III score alone was not able to discriminate among the three groups of subjects (Table 1). The finding that significant differences in the $\mathrm{CBFv}$ response were obtained for this task (Fig. 2, Table 3) is encouraging and highlights the potential of this novel approach. Finally, we focused on a task involving speech as this presents particular feasibility challenges for TCD-based NVC studies, given the potential interference of speech with the Doppler signal quality and parallel changes in $\mathrm{ETCO}_{2}$, as reflected by the temporal patterns of $\mathrm{ETCO}_{2}$ in Fig. 1.

Despite the very promising results obtained for feasibility and sensitivity with the selected task, caution is needed at this stage regarding the potential performance for the other 19 paradigms. Indeed, further studies, and detailed data analyses, will be needed to identify those that would optimize the clinical benefits of adding TCD as an adjunct to the standard ACE-III examination.

\section{Neurovascular coupling response}

Despite demonstrating a difference in stimulation between groups, in this population, language was largely well preserved in the AD and MCI groups, with no differences on ACE-III score, Table 1. This may suggest that the differences in $\mathrm{CBFv}$ change to the language task across groups are more likely 
due to the changes in peripheral parameters, rather than stimulation. However, these results may also indicate that reduced $\mathrm{CBFv}$ response is a more sensitive marker than clinical cognitive testing and that subtle changes in CBFv may be apparent before clinical deficits are detectable. This is supported by the sustained differences in $\mathrm{CBFv}$ response at $\mathrm{T} 3$, despite a lack of change in the peripheral parameters at this time point.

Changes in peripheral parameters have previously been reported in a number of task activation studies, where sympathetic activation can result in rises in HR and MAP, and hyperventilation or breath holding can result in changes in $\mathrm{ETCO}_{2}[14,25]$. Cardioinhibitory effects, due to parasympathetic activation, of AChEIs have been documented previously [26, 27]. In this cohort, AChEI use was significantly higher among the AD group, and could account for the blunted HR response to the language task. A number of recent studies have demonstrated that changes in CBFv are likely to be as a result of changes occurring at the metabolic or myogenic level in response to cognitive [28-30], or motor [31] stimulation. This challenges the interpretation that $\mathrm{CBFv}$ changes are purely a reflection of changes in MAP [23].

\section{Cognitive impairment and vessel function}

Impaired cerebral blood flow occurs early in patients with both MCI and dementia [5, 6]. The two-hit hypothesis describes a cascade of pathological events, initiated and propagated by impaired $\mathrm{CBF}$, resulting in progressive amyloid accumulation and tau hyperphosphorylation [5, 32]. Amyloid accumulation not only damages neurons directly, but also damages the structural integrity of blood vessels, and induces vasoconstriction, thus worsening cerebral hypoperfusion [5, 32]. Furthermore, amyloid accumulation results in blood-brain barrier dysfunction, protein leakage, and further neuronal toxicity $[5,32,33]$. Atherosclerosis in the circle of Willis is found more commonly among those with AD dementia, than non-AD dementia [32]. Despite these findings at a microvascular level, studies of vessel function in dementia and MCI remain mixed $[6,7,9]$. In a recent study, despite lower resting CBFv in dementia, cerebral autoregulation was better in dementia compared to healthy controls [34]. However, while dynamic cerebral autoregulation appears to remain intact, a number of meta-analyses have consistently shown lower resting flow velocities, higher vessel resistance, and lower vasoreactivity in cognitive impairment $[6,7,9]$. Fewer studies have examined NVC in dementia, and these are outlined in more detailed below. Thus, NVC may be a more useful maker of cognitive impairment than dynamic $\mathrm{CBF}$ autoregulation.

\section{Results in context of existing literature}

Few studies have employed TCD as a method by which to investigate NVC in dementia $[6,7,9$, 35]. Certainly, recent reviews and meta-analyses have consistently demonstrated lower resting CBF in both $\mathrm{AD}$ and $\mathrm{VaD}$ sub-types of dementia $[6,7,9,35$, 36]. Furthermore, markers of vessel resistance (pulsatility index, cerebrovascular resistance index) are higher among those with both dementia sub-types and the ability of the cerebrovasculature to respond to changes in $\mathrm{CO}_{2}$ is lost (vasoreactivity) $[6,7,9$, 36]. Furthermore, $\mathrm{VaD}$ and $\mathrm{AD}$ can be discriminated from one another on the basis of pulsatility index and vasoreactivity [7].

No previous studies have measured NVC in response to a language paradigm in cognitive impairment from the ACE-III by TCD. Two TCD studies examined $\mathrm{CBFv}$ responses in the posterior cerebral artery during visual stimulation, where those with $\mathrm{VaD}$ could be discriminated from $\mathrm{AD}$ and healthy controls $[16,37]$. These findings suggest intact occipital function in early $\mathrm{AD}[16,37]$. The results presented here, suggest that $\mathrm{CBFv}$ responses to language stimulation may be detected at an earlier stage than visual stimulation, and therefore may be a better discriminator of cognitive impairment from healthy aging. In a study by Matteis et al., a verbal fluency task produced distinctly lateralized rises in $\mathrm{CBFv}$ in the healthy control group; however, bilateral rises were seen in both patient groups, with loss of hemisphere selectively in the $\mathrm{CBFv}$ response [17]. The bilateral rise in $\mathrm{CBFv}$ is likely to represent a compensatory mechanism to cope with rising cognitive demands resulting from declining cognitive ability $[38,39]$. This phenomenon has also been demonstrated in older adults [38, 39], and those with MCI and early dementia [40]. In keeping with findings of Matteis et al. [17], Yeung et al. also found loss of hemispheric specialization, using a category fluency task [41]. Interestingly, this was detectable as early as the MCI stage, and was more pronounced in those with amnestic sub group MCI [41]. In contrast to these studies, we did not find a significant difference in hemispheric lateralization between or within groups. Although left handers might have a tendency to less 
lateralization in response to cognitive testing [14], the fact that our population included only two left handed participants in each group is unlikely to explain the lack of a significant hemispheric lateralization within groups. On analyzing data for right-handed participants only $(n=25)$, there were no differences in baseline $\mathrm{CBFv}$ or peripheral parameter data, nor in peak percentage change, indicating that left-handed individuals did not significantly affect the results of this feasibility study. In keeping with our findings here, a number of MRI, PET, and SPECT studies have demonstrated correlation between language deficits in MCI and $\mathrm{AD}$, and regional hypoperfusion or hypometabolism [42-45]. In addition, recent fMRI studies report reduced activation during language processing in $\mathrm{MCI}$ and $\mathrm{AD}$, as demonstrated here [44-47], but also compensatory hyperactivation in MCI groups [45, 48]. In an fMRI study by Vandenbulcke et al., amnestic MCI patients with preserved language function, demonstrated reduced activation in the posterior temporal lobes compared to controls during a word reading task [46]. Taken together, these results suggest that $\mathrm{CBF}$ responses to language tasks can be detected prior to the appearance of clinical deficits, and thus could provide an early imaging biomarker of cognitive decline.

\section{Limitations}

Measurement of resting flow velocities relies on the assumption that the vessel diameter remains constant and therefore $\mathrm{CBFv}$ can be measured to approximate for CBF [10]. This study reports a high window failure rate $(\sim 24 \%)$, however this is consistent with other studies of cognitive impairment and older adults [7, 49]. The sample size studied here was relatively small, and results need to be interpreted in this context. In addition, diagnostic classification was found to be complex, we therefore employed a consensus approach to classifying participants in this study. Due to the window failure rate, a number of participants were not able to be matched on age and sex, and were therefore excluded from this analysis.

The MCA supplies approximately $80 \%$ of the cerebral cortex [14] and, while we can achieve high temporal resolution by TCD measured CBFv change $[10,14]$, we cannot map this regionally to determine specifically where these changes are occurring.

In terms of the population studied, there was a relatively higher window failure rate among females, and they are therefore under-represented in this study. As expected, the mean age was relatively high in this study, with more volunteers recruited with older onset cognitive impairment. The majority of the cohort were Caucasian, and few were left handed. The results may therefore not be generalizable to the wider population. In addition, for this study it was assumed that left handed individuals would lateralize to the right hemisphere in terms of dominance. However, this is not always the cases, with approximately one quarter of left handed individuals demonstrating atypical lateralization patterns [50]. A more complex classification of hemispheric dominance can instead be achieved through calculation of the laterality index based on relative hemispheric response to cognitive testing [51].

In previous studies of task activation, multiple cycles of activation have been utilized to improve signal-to-noise ratios [36], but we have previously demonstrated that peak changes in CBFv are obtainable with one cycle of task activation in healthy subjects, thus limiting accommodation of responses through repeated trials [22]. One important finding of the current study though, is the demonstration that a single cycle of activation is also feasible in patients with $\mathrm{MCI}$ and $\mathrm{AD}$ and leads to good quality measured responses. In this study, TCD alone was used, with no method of measuring neuronal activation directly, such as electroencephalography (EEG). Future studies can consider the combination of these techniques to measure flow and neuronal activity simultaneously.

\section{Implications of the findings and further work}

This study has demonstrated that TCD-measured task activation to a language task taken from a clinical assessment tool (ACE-III) is feasible in a cognitively impaired population. The protocol was acceptable to patients and we were able to achieve measures of change in $\mathrm{CBFv}, \mathrm{MAP}, \mathrm{HR}$, and, $\mathrm{ETCO}_{2}$. This study has highlighted a number of key areas that require further consideration for future studies of TCD in dementia.

Specifically, further studies with larger sample sizes, examining a range of paradigms within the sub-domains of the ACE-III are required to identify if these trends are seen across the different cognitive domains, or isolated to individual tasks. Future studies should ideally measure concomitant changes in MAP, $\mathrm{HR}$, and $\mathrm{ETCO}_{2}$ alongside $\mathrm{CBFv}$ response. The use of more complex dynamic multivariate modelling, examining the relative contributions of MAP, $\mathrm{HR}, \mathrm{ETCO}_{2}$, and stimulation to the total $\mathrm{CBFv}$ response, would allow for a more accurate and com- 
prehensive assessment of NVC in TCD studies of task activation in dementia. Studies with a broader population, to examine changes in female and ethnic minority groups would allow results to be more generalizable to the wider population. The inclusion of participants with $\mathrm{VaD}$ would improve our understanding of the changes in NVC occurring comparatively in dementia sub-types.

\section{Conclusions}

There is a growing body of evidence that vascular dysfunction is an important pathological mechanism implicated in the development of $\mathrm{AD}$, in addition to $\mathrm{VaD}$. This warrants further investigation both as a biomarker of early disease, but also for novel therapeutic approaches. TCD measured CBFv changes are feasible in a cognitively impaired population and remains a promising approach to studying NVC changes associated with aging and dementia.

\section{ACKNOWLEDGMENTS}

TGR is an NIHR Senior Investigator. LCB is an NIHR Academic Clinical Fellow.

\section{CONFLICTS OF INTEREST}

The authors have no conflict of interest to report.

\section{REFERENCES}

[1] Prince M, Wimo A, Guerchet M, Ali GC, Wu Y, Prina M (2015) World Alzheimer Report 2015 The global impact of dementia. Alzheimer's Disease International, London, UK, pp. 1-87.

[2] Petersen RC (2004) Mild cognitive impairment as a diagnostic entity. J Intern Med 256, 183-194.

[3] de la Torre JC (2012) Cerebral hemodynamics and vascular risk factors: Setting the stage for Alzheimer's disease. J Alzheimers Dis 32, 553-567.

[4] Hays CC, Zlatar ZZ, Wierenga CE (2016) The utility of cerebral blood flow as a biomarker of preclinical Alzheimer's disease. Cell Mol Neurobiol 36, 167-179.

[5] Zlokovic BV (2011) Neurovascular pathways to neurodegeneration in Alzheimer's disease and other disorders. Nat Rev Neurosci 12, 723-738.

[6] Beishon L, Haunton VJ, Panerai RB, Robinson TG (2017) Cerebral hemodynamics in mild cognitive impairment: A systematic review. J Alzheimers Dis 59, 369-385.

[7] Keage HA, Churches OF, Kohler M, Pomeroy D, Luppino R, Bartolo ML, Elliott S (2012) Cerebrovascular function in aging and dementia: A systematic review of transcranial Doppler studies. Dement Geriatr Cogn Dis Extra 2, 258-270.
[8] Tomek A, Urbanova B, Hort J (2014) Utility of transcranial ultrasound in predicting Alzheimer's disease risk. $J$ Alzheimers Dis 42, S365-S374.

[9] Sabayan B, Jansen S, Oleksik AM, van Osch MJ, van Buchem MA, van Vliet P, de Craen AJ, Westendorp RG (2012) Cerebrovascular hemodynamics in Alzheimer's disease and vascular dementia: A meta-analysis of transcranial Doppler studies. Ageing Res Rev 11, 271-277.

[10] Panerai RB (2009) Transcranial Doppler for evaluation of cerebral autoregulation. Clin Auton Res 19, 197-211.

[11] van Beek AH, Claassen JA, Rikkert MG, Jansen RW (2008) Cerebral autoregulation: An overview of current concepts and methodology with special focus on the elderly. J Cereb Blood Flow Metab 28, 1071-1085.

[12] Aaslid R (1987) Visually evoked dynamic blood flow response of the human cerebral circulation. Stroke 18, 771775.

[13] Moody M, Panerai RB, Eames PJ, Potter JF (2005) Cerebral and systemic hemodynamic changes during cognitive and motor activation paradigms. Am J Physiol Regul Integr Comp Physiol 288, R1581-1588.

[14] Stroobant N, Vingerhoets G (2000) Transcranial Doppler ultrasonography monitoring of cerebral hemodynamics during performance of cognitive tasks: A review. Neuropsychol Rev 10, 213-231.

[15] Phillips AA, Chan FH, Zheng MM, Krassioukov AV, Ainslie PN (2016) Neurovascular coupling in humans: Physiology, methodological advances and clinical implications. J Cereb Blood Flow Metab 36, 647-664.

[16] Asil T, Uzuner N (2005) Differentiation of vascular dementia and Alzheimer disease: A functional transcranial Doppler ultrasonographic study. J Ultrasound Med 24, 1065-1070.

[17] Matteis M, Silvestrini M, Troisi E, Bragoni M, Vernieri F, Caltagirone C (1998) Cerebral hemodynamic patterns during stimuli tasks in multi-infarct and Alzheimer types of dementia. Acta Neurol Scand 97, 374-380.

[18] Rosengarten B, Paulsen S, Molnar S, Kaschel R, Gallhofer B, Kaps M (2007) Activation-flow coupling differentiates between vascular and Alzheimer type of dementia. J Neurol Sci 257, 149-154.

[19] Ferris SH, Farlow M (2013) Language impairment in Alzheimer's disease and benefits of acetylcholinesterase inhibitors. Clin Interv Aging 8, 1007-1014.

[20] Oldfield RC (1971) The assessment and analysis of handedness: The Edinburgh inventory. Neuropsychologia 9, 97-113.

[21] Beishon L, Williams CAL, Panerai RB, Robinson TG, Haunton VJ (2017) Reproducibility of task activation using the Addenbrooke's cognitive examination in healthy controls: A functional Transcranial Doppler ultrasonography study. J Neurosci Methods 291, 131-140.

[22] Beishon LC, Williams CAL, Panerai RB, Robinson TG, Haunton VJ (2018) The assessment of neurovascular coupling with the Addenbrooke's Cognitive Examination: A functional Transcranial Doppler Ultrasonographic Study. J Neurophysiol 119, 1084-1094.

[23] Panerai RB, Moody M, Eames PJ, Potter JF (2005) Cerebral blood flow velocity during mental activation: Interpretation with different models of the passive pressure-velocity relationship. J Appl Physiol (1985) 99, 2352-2362.

[24] Williams CAL, Panerai RB, Robinson TG, Haunton VJ (2017) Transcranial Doppler ultrasonography in the assessment of neurovascular coupling responses to cognitive 
examination in healthy controls: A feasibility study. $\mathrm{J} \mathrm{Neu}$ rosci Methods 284, 57-62.

[25] Silvestrini M, Cupini LM, Matteis M, Troisi E, Caltagirone C (1994) Bilateral simultaneous assessment of cerebral flow velocity during mental activity. J Cereb Blood Flow Metab 14, 643-648.

[26] Jackson S, Ham RJ, Wilkinson D (2004) The safety and tolerability of donepezil in patients with Alzheimer's disease. Br J Clin Pharmacol 58(Suppl 1), 1-8.

[27] Bordier P, Garrigue S, Lanusse S, Margaine J, Robert F, Gencel L, Lafitte A (2006) Cardiovascular effects and risk of syncope related to donepezil in patients with Alzheimer's disease. CNS Drugs 20, 411-417.

[28] Tan CH, Low KA, Schneider-Garces N, Zimmerman B, Fletcher MA, Maclin EL, Chiarelli AM, Gratton G, Fabiani M (2016) Optical measures of changes in cerebral vascular tone during voluntary breath holding and a Sternberg memory task. Biol Psychol 118, 184-194.

[29] Szirmai I, Amrein I, Palvolgyi L, Debreczeni R, Kamondi A (2005) Correlation between blood flow velocity in the middle cerebral artery and EEG during cognitive effort. Brain Res Cogn Brain Res 24, 33-40.

[30] Boban M, Crnac P, Junakovic A, Malojcic B (2014) Hemodynamic monitoring of middle cerebral arteries during cognitive tasks performance. Psychiatry Clin Neurosci 68, 795-803.

[31] Salinet AS, Robinson TG, Panerai RB (2013) Cerebral blood flow response to neural activation after acute ischemic stroke: A failure of myogenic regulation? J Neurol 260, 2588-2595.

[32] Liu W, Wong A, Law AC, Mok VC (2015) Cerebrovascular disease, amyloid plaques, and dementia. Stroke 46, 14021407.

[33] Montagne A, Zhao Z, Zlokovic BV (2017) Alzheimer's disease: A matter of blood-brain barrier dysfunction? J Exp Med 214, 3151-3169.

[34] de Heus RAA, de Jong DLK, Sanders ML, van Spijker GJ, Oudegeest-Sander MH, Hopman MT, Lawlor BA, Olde Rikkert MGM, Claassen JAHR (2018) Dynamic regulation of cerebral blood flow in patients with Alzheimer disease. Hypertension 72, 139-150.

[35] Claassen JA, Zhang R (2011) Cerebral autoregulation in Alzheimer's disease. J Cereb Blood Flow Metab 31, 15721577.

[36] Malojcic B, Giannakopoulos P, Sorond FA, Azevedo E, Diomedi M, Oblak JP, Carraro N, Boban M, Olah L, Schreiber SJ, Pavlovic A, Garami Z, Bornstein NM, Rosengarten B (2017) Ultrasound and dynamic functional imaging in vascular cognitive impairment and Alzheimer's disease. BMC Med 15, 27.

[37] Rosengarten B, Kaps M (2010) A simultaneous EEG and transcranial Doppler technique to investigate the neurovascular coupling in the human visual cortex. Cerebrovasc Dis 29, 211-216.

[38] Cabeza R (2002) Hemispheric asymmetry reduction in older adults: The HAROLD model. Psychol Aging 17, 85-100.

[39] Berlingeri M, Danelli L, Bottini G, Sberna M, Paulesu E (2013) Reassessing the HAROLD model: Is the hemispheric asymmetry reduction in older adults a special case of compensatory-related utilisation of neural circuits? Exp Brain Res 224, 393-410.

[40] Dickerson BC, Sperling RA (2008) Functional abnormalities of the medial temporal lobe memory system in mild cognitive impairment and Alzheimer's disease: Insights from functional MRI studies. Neuropsychologia 46, 16241635.

[41] Yeung MK, Sze SL, Woo J, Kwok T, Shum DH, Yu R, Chan AS (2016) Altered frontal lateralization underlies the category fluency deficits in older adults with mild cognitive impairment: A near-infrared spectroscopy study. Front Aging Neurosci 8, 59.

[42] Leeuwis AE, Benedictus MR, Kuijer JPA, Binnewijzend MAA, Hooghiemstra AM, Verfaillie SCJ, Koene T, Scheltens P, Barkhof F, Prins ND, van der Flier WM (2017) Lower cerebral blood flow is associated with impairment in multiple cognitive domains in Alzheimer's disease. Alzheimers Dement 13, 531-540.

[43] Takahashi M, Oda Y, Okubo T, Shirayama Y (2017) Relationships between cognitive impairment on ADAS-cog and regional cerebral blood flow using SPECT in late-onset Alzheimer's disease. J Neural Transm (Vienna) 124, 11091121.

[44] Bosch B, Bartres-Faz D, Rami L, Arenaza-Urquijo EM, Fernandez-Espejo D, Junque C, Sole-Padulles C, SanchezValle R, Bargallo N, Falcon C, Molinuevo JL (2010) Cognitive reserve modulates task-induced activations and deactivations in healthy elders, amnestic mild cognitive impairment and mild Alzheimer's disease. Cortex 46, 451461.

[45] Li H-J, Hou X-H, Liu H-H, Yue C-L, He Y, Zuo X-N (2015) Toward systems neuroscience in mild cognitive impairment and Alzheimer's disease: A meta-analysis of 75 fMRI studies. Hum Brain Mapp 36, 1217-1232.

[46] Vandenbulcke M, Peeters R, Dupont P, Van Hecke P, Vandenberghe $\mathrm{R}$ (2007) Word reading and posterior temporal dysfunction in amnestic mild cognitive impairment. Cereb Cortex 17, 542-551.

[47] Olichney JM, Taylor JR, Chan S, Yang JC, Stringfellow A, Hillert DG, Simmons AL, Salmon DP, Iragui-Madoz V, Kutas M (2010) fMRI responses to words repeated in a congruous semantic context are abnormal in mild Alzheimer's disease. Neuropsychologia 48, 2476-2487.

[48] Lenzi D, Serra L, Perri R, Pantano P, Lenzi GL, Paulesu E, Caltagirone C, Bozzali M, Macaluso E (2011) Single domain amnestic MCI: A multiple cognitive domains fMRI investigation. Neurobiol Aging 32, 1542-1557.

[49] Wijnhoud AD, Franckena M, van der Lugt A, Koudstaal PJ, Dippel ED (2008) Inadequate acoustical temporal bone window in patients with a transient ischemic attack or minor stroke: Role of skull thickness and bone density. Ultrasound Med Biol 34, 923-929.

[50] Szaflarski JP, Binder JR, Possing ET, McKiernan KA, Ward BD, Hammeke TA (2002) Language lateralization in lefthanded and ambidextrous people: FMRI data. Neurology 59, 238-244.

[51] Knecht S, Deppe M, Ringelstein EB, Wirtz M, Lohmann H, Drager B, Huber T, Henningsen H (1998) Reproducibility of functional transcranial Doppler sonography in determining hemispheric language lateralization. Stroke 29, 1155-1159. 